\title{
Segurança e aplicação da plataforma vibratória em pacientes hospitalizados: uma revisão sistemática
}

\section{Safety and application of the vibratory platform in hospitalized patients: a systematic review}

\author{
Renata Ferreira de Moura' ${ }^{1}$ (1) \\ Alan Carlos Nery dos Santos ${ }^{2}$ (1) \\ Ramon Martins Barbosa ${ }^{3}$ (1) \\ Bruno Prata Martinez 4 (1) \\ Vinícius Afonso Gomes 5 (1)
}

\begin{abstract}
${ }^{1}$ Autora para correspondência. Universidade Salvador (Feira de Santana). Bahia, Brasil. renataferreirademoura@outlook.com.br ²Universidade Salvador (Feira de Santana), Escola Bahiana de Medicina e Saúde Pública (Salvador). Bahia, Brasil. allannery.santos@hotmail.com,

${ }^{3}$ Universidade Salvador (Feira de Santana). Bahia, Brasil. ramonmartinsbarbosa@hotmail.com ${ }^{4}$ Universidade Federal da Bahia (Salvador), Universidade do Estado da Bahia (Salvador). Bahia, Brasil. Bahia, Brasil. bruno.martinez@ufba.br 5Universidade Salvador (Feira de Santana), Escola Bahiana de Medicina e Saúde Pública (Salvador), Centro Universitário UniRuy Wyden (Salvador).
\end{abstract}

Bahia, Brasil. vinifisioterapia@yahoo.com.br
RESUMO | INTRODUÇÃo: Um curto período de hospitalização, se acompanhado de imobilidade, pode ser capaz de promover declínio das funções musculoesqueléticas, gerando impacto negativo na funcionalidade e qualidade de vida. Com isso, tem-se tornado cada vez mais comum a adoção de estratégias terapêuticas como o uso da plataforma vibratória (PV). OBJETIVO: Sistematizar evidências sobre a funcionalidade e a segurança da aplicação da PV em pacientes adultos hospitalizados. MÉTODOS: Revisão sistemática, registrada na PROSPERO com código CRD42019119672. Desenvolvida nas bases: LILACS, SciELO, MedLine/PubMed, EBSCOhost e PEDro. Descritores e palavras-chave: "Whole body vibration", "Intensive Care Units", "hospitalization", "muscle strenght", e "functional capacity". Incluídos estudos que analisaram os efeitos e a segurança da aplicação da PV em pacientes adultos hospitalizados. A qualidade metodológica foi avaliada através da escala Downs and Black. RESULTADOS: Incluídos 2 estudos, um ensaio clínico randomizado e outro estudo de intervenção controlado. A amostra variou entre 24 e 40 sujeitos, de ambos os sexos, média de idade $52 \pm 4$ anos, com diagnóstico de DPOC e condições variadas. Houve uma meIhora na distância percorrida no teste de caminhada de seis minutos e diminuição no tempo do teste de sentar e levantar, aumento dos níveis de irisina e melhora na qualidade de vida, em relação aos parâmetros dos sinais vitais não teve alterações significativas. O escore metodológico foi em média 16. CONCLUSÃo: Os resultados indicam que a PV parece ser viável e segura, podendo trazer efeitos favoráveis na funcionalidade para o tratamento em pacientes adultos hospitalizados, sendo uma alternativa para a reabilitação de forma precoce.

PALAVRAS-CHAVE: Unidade de Terapia Intensiva. Hospitalização. Força muscular.
ABSTRACT | INTRODUCTION: A short period of hospitalization, if accompanied by immobility, may be able to promote a decline in musculoskeletal functions, generating a negative impact on functionality and quality of life. As a result, the adoption of therapeutic strategies such as the use of the vibrating platform (PV) has become increasingly common. OBJECTIVE: To systematize evidence on the functionality and safety of the application of PV in hospitalized adult patients. METHODS: Systematic review, registered at PROSPERO with code CRD42019119672. Developed in the bases: LILACS, SciELO, MedLine / PubMed, EBSCOhost and PEDro. Keywords and keywords: "Whole body vibration", "Intensive Care Units", "hospitalization", "muscle strength", and "functional capacity". Included studies that analyzed the effects and safety of the application of PV in hospitalized adult patients. Methodological quality was assessed using the Downs and Black scale. RESULTS: Included 2 studies, a randomized clinical trial and another controlled intervention study. The sample varied between 24 and 40 subjects, of both sexes, mean age $52 \pm 4$ years, with a diagnosis of COPD and varied conditions. There was an improvement in the distance covered in the six-minute walk test and a decrease in the time of the sit and stand test, an increase in irisin levels and an improvement in quality of life, in relation to the vital signs parameters, there was no significant change. The methodological score was on average 16. CONCLUSION: The results indicate that PV seems to be viable and safe, and may have favorable effects on functionality for treatment in hospitalized adult patients, being an alternative for early rehabilitation.

KEYWORDS: Intensive Care Units. Hospitalization. Muscle strength. 


\section{Introdução}

No ambiente hospitalar a assistência promovida pelos profissionais da reabilitação objetiva recuperar e/ou preservar as condições clínico-funcionais, bem como, devolver a capacidade de autonomia para atividades de vida diária básica e instrumental dos pacientes o mais próximo do seu estado prévio a internação ${ }^{1}$. Segundo a North American Nursing Diagnosis Association, o imobilismo apresenta-se como um notável fator de limitações funcionais no indivíduo hospitalizado, sendo caracterizado como um estado de restrição do movimento² .

Esta imobilidade prolongada promove efeitos deletérios para o sistema musculoesquelético por diversos mecanismos que aumentam a proteólise e reduzem o trofismo muscular ${ }^{3}$. Somado a isso, foi demonstrado que mesmo um curto período de hospitalização, se acompanhado de acinesia, pode ser capaz de promover declínio da potência dos membros, gerando impacto negativo na funcionalidade ${ }^{4}$.

A persistência e a gravidade da fraqueza muscular geram ainda como fatores agravantes, maior índice de morbidade e mortalidade, além de aumento dos custos relacionados à assistência em saúde ${ }^{5}$. Entretanto, existem estratégias para combater essa inatividade, como a mobilização precoce. Esta prática pode reduzir as adaptações desfavoráveis e as complicações geradas pela hipomobilidade no leito, otimizando assim a condição motora daqueles que ao trabalho de mobilidade são expostos ${ }^{6}$.

São várias as estratégias de mobilização pelas quais é possível manter, minimizar e/ou aumentar a capacidade funcional. Dentre elas podemos destacar a retirada do paciente do leito através de caminhadas controladas; sedestação em poltronas; utilização de exercícios neuromusculares; eletroestimulação funcional; uso de prancha ortostática, cicloergômetro dentre outras ${ }^{7-11}$. No entanto, a escolha da terapia adequada depende de fatores como o nível de colaboração, condição clínica e hemodinâmica do sujeito, além da disponibilidade de recursos da unidade em questão ${ }^{12}$.
Além das terapias para mobilização propostas, temse tornado cada vez mais comum o uso da plataforma vibratória (PV) como uma ferramenta alternativa para combater a hipocinesia, uma vez que sua aplicação produz oscilações sinusoidais que promovem ativação neuromuscular ${ }^{13}$. Logo, tem sido demonstrado que a exposição às forças aplicadas sobre os tendões e músculos através da $P V$, tende a melhorar o desempenho muscular ${ }^{14}$. A PV por estimular efetivamente o sistema neuromuscular de indivíduos é responsável por melhorar a capacidade cardiorrespiratória e força muscular ${ }^{15}$. Nos pacientes críticos essa estratégia estimula os músculos e melhora o metabolismo muscular, por isso pode ter o potencial de prevenir e/ou tratar a fraqueza muscular ${ }^{16}$.

Apesar dos benefícios já relatados da aplicação da PV em outros desfechos em diferentes populações, ainda não é bem definido a sua aplicabilidade no ambiente hospitalar como potencial terapêutica para prevenir o agravamento dos efeitos deletérios durante a hospitalização. Dessa forma, a presente pesquisa teve como objetivo avaliar os efeitos na funcionalidade e a segurança da aplicação da plataforma vibratória em pacientes adultos hospitalizados.

\section{Métodos}

\section{Delineamento}

Trata-se de uma revisão sistemática, desenhada com base nos critérios estabelecidos pelo guideline Preferred Reporting Items for Systematic Reviews and Meta-Analyses - PRISMA ${ }^{17}$, para responder à pergunta de investigação baseados na estratégia PICO (População, Intervenção, Comparação, Desfechos): em pacientes adultos hospitalizados o uso da plataforma vibratória é segura e/ou adiciona benefícios na funcionalidade quando comparada a uma intervenção controle? O protocolo desta revisão está registrado na base de dados para estudo de revisão sistemática PROSPERO, sob registro CRD42019119672. 


\section{Critérios de elegibilidade}

Foram incluindo artigos originais de intervenção, que compararam grupos PV versus grupos controle, que analisaram os efeitos ou, a segurança de um programa de fisioterapia com PV em pacientes adultos ( $\geq 18$ anos), de ambos os sexos, hospitalizados. Não foram realizadas restrições quanto ao tempo de publicação dos estudos ou ao idioma.

\section{Critérios de não inclusão}

Estudos com pacientes em ambiente ambulatorial, ou, centros de reabilitação, protocolos de estudos, estudos não indexados nas bases de dados, além de estudos pilotos.

\section{Estratégia de busca}

As buscas por potenciais estudos foram realizadas entre o período de agosto a dezembro de 2018 por dois revisores independentes, sendo que se houvesse alguma divergência, um terceiro revisor seria solicitado. Uma nova busca foi realizada no mês de agosto de 2020 para possíveis atualizações de novos estudos. Durante esse período foram consultadas as bases de dados LILACS, SciELO, MedLine/PubMed, EBSCOhost e PEDro. A triagem dos estudos foi realizada por meio dos descritores e palavras-chave: "Whole body vibration", "Intensive Care Units", "hospitalization", "muscle strenght", e "functional capacity". O termo "Whole body vibration" e "Functional Capacity" por não estarem indexados no Descritores em Ciências da Saúde (DECS) foi definido a partir de consulta com especialista na área, dessa forma foram realizadas várias estratégias de cruzamento, assim detalhados no Quadro 1, para evitar a perca de estudos na área. Os operadores booleanos AND e OR foram utilizados para estratégias de cruzamento.

\section{Seleção de estudos}

O processo de triagem dos artigos se deu inicialmente por meio da leitura dos títulos e resumos. Em seguida, foram excluídos os estudos que não atendiam aos critérios de elegibilidade. Assim, os trabalhos científicos que atenderam aos critérios estabelecidos foram recuperados para leitura do texto na íntegra, nova avaliação quanto a elegibilidade e extração dos desfechos de interesse da presente revisão sistemática.

Quadro 1. Cruzamentos realizados de acordo com as bases de dados selecionadas

\begin{tabular}{|c|c|}
\hline BASE DE DADOS & "CRUZAMENTOS" \\
\hline \multirow{3}{*}{$\begin{array}{l}\text { LILACS, SciELO, } \\
\text { EBSCOhost }\end{array}$} & “Whole Body Vibration” AND “Hospitalization” \\
\hline & "Whole Body Vibration" AND "Intensive Care Units" \\
\hline & $\begin{array}{l}\text { "Whole Body Vibration" AND "Muscle Strenght" OR "Functional Capacity" AND } \\
\text { "Hospitalization" }\end{array}$ \\
\hline \multirow{3}{*}{ Pubmed/MEDLINE } & “Whole Body Vibration” AND “Hospitalization” \\
\hline & "Whole Body Vibration" AND "Intensive Care Units" \\
\hline & "Whole Body Vibration" AND “Muscle Strenght" AND "Functional Capacity” \\
\hline \multirow{4}{*}{ PEDro } & "Whole Body Vibration" AND “Hospitalization" \\
\hline & "Whole Body Vibration" AND "Intensive Care Units" \\
\hline & "Whole Body Vibration" AND "Muscle Strenght" \\
\hline & “Whole Body Vibration" AND "Functional Capacity" \\
\hline
\end{tabular}




\section{Extração de dados}

Os dados dos estudos foram extraídos por dois revisores de forma independente e resumidos em tabelas. Esses dados incluem o desenho do estudo, as características das amostras e das intervenções, o grupo controle, os desfechos analisados e os principais resultados obtidos.

\section{Desfechos de interesse}

A funcionalidade foi analisada como uma melhora na distância percorrida no teste de caminhada de 6 minutos e na qualidade de vida, além do aumento da força muscular. A segurança foi avaliada de acordo com os parâmetros clínicos dos pacientes como sinais vitais e a incidência de efeitos adversos.

\section{Qualidade do estudo e risco de viés}

Para a análise metodológica dos artigos que atenderam aos critérios de inclusão, foi utilizada a escala Downs and Black ${ }^{18}$ pontuada em 27 questões que se dividiam em 5 domínios: forma de reportar resultados (10 itens), validade externa (3 itens), validade interna - vieses ( 7 itens), fatores de confusão ( 6 itens) e poder do estudo ( 1 item). Para cada questão, aplicou-se o escore 0 , caso o artigo não fosse pertinente ao que se está avaliando, e o escore 1 quando apresentava resposta positiva ao requisito, com exceção do item 5 que tem pontuação máxima de 2 . 0 item 27 foi modificado conforme usado em outros estudos ${ }^{19,20}$, onde o escore que ia de 0 a 5 , passa a ser 0 a 1 , onde 0 se 0 artigo não apresentasse o cálculo do tamanho amostral, e 1 se apresentasse.

\section{Síntese dos resultados}

O presente estudo não cabe uma síntese com metanálise devido aos estudos encontrados avaliarem desfechos diferentes.

\section{Resultados}

\section{Seleção de estudos}

Foram identificados 396 estudos, dos quais 40 foram excluídos por apresentarem duplicidade nas bases de dados. Após a eliminação dos estudos duplicados e exclusões a partir da análise de títulos e resumos, 15 artigos foram considerados elegíveis para leitura do texto na íntegra. Posteriormente, 13 artigos foram excluídos por não cumprirem os critérios de elegibilidade. Dessa forma foram incluídos apenas 2 estu$\operatorname{dos}^{21,22}$ na presente revisão. A Figura 1 sintetiza o processo de seleção dos estudos que compõe o escopo desta revisão sistemática. 


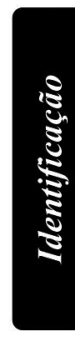

Relatos identificados através de pesquisas nas bases de dados: Pubmed/Medline, EBSCO, SCielo, LILACS, PEDro $\mathrm{N}=396$

Relatos adicionais identificados através de outras fontes $\mathrm{N}=0$

341 relatos excluídos após a leitura dos títulos e resumos

Relatos artigos em texto completo avaliados $\mathrm{N}=15$

Artigos incluídos nesta revisão $\mathrm{N}=2$

13 artigos excluídos após a leitura do texto na íntegra

Programa de reabilitação ambulatorial

$$
(n=5)
$$

Centro de reabilitação $(n=4)$

Não eram hospitalizados $(n=2)$

Protocolo de estudo $(n=1)$

Estudos de intervenção não controlado $(n=1)$

\section{Análise da Qualidade Metodológica dos estudos}

Os trabalhos foram avaliados quanto à sua qualidade metodológica através dos critérios da escala Downs and Black subdivididos em 5 domínios, utilizada para avaliar tanto estudos randomizados quanto não randomizados. O escore poderia variar entre 0 a 28, não tendo pontuação mínima, assim quanto maior o número de itens abrangidos por cada estudo, maior foi considerada sua qualidade metodológica.

A média do escore metodológico foi de 16 pontos. O estudo que obteve maior pontuação foi o de Greulich et al. ${ }^{21}$ em comparação com o de Boeselt et al. ${ }^{22}$ Dentre os critérios da avaliação da qualidade metodológica pela Downs and Black as principais limitações foram em relação a falta de "cegamento"; não descrever claramente os critérios de elegibilidade; não relatar ajustes para fatores de confusão e o baixo poder estatístico para poder detectar efeitos importantes, já que nenhum estudo fez cálculo de tamanho amostral ou poder, além das variáveis de desfechos distintas dos estudos. (Tabela 1) 
Tabela 1. Dados sobre a qualidade dos estudos por domínio

\begin{tabular}{|c|c|c|}
\hline & Greulich et al.. ${ }^{21}$ & Boeselt et al. ${ }^{22}$ \\
\hline & Escore & Escore \\
\hline Reportar resultados & $8 / 11$ & $8 / 11$ \\
\hline Validade externa & $2 / 3$ & $1 / 3$ \\
\hline Viés & $6 / 7$ & $4 / 7$ \\
\hline Viés de seleção & $3 / 6$ & $0 / 6$ \\
\hline Poder & $0 / 1$ & $0 / 1$ \\
\hline & \multicolumn{2}{|c|}{ Escore total Downs and Black } \\
\hline & $19 / 28$ & $13 / 28$ \\
\hline
\end{tabular}

1. Objetivo / Hipótese de estudo; 2. Desfechos principais a serem avaliados na introdução ou métodos; 3. Características dos pacientes incluídos, critérios de elegibilidade; 4. Intervenções de interesse descritas 5. Distribuição dos fatores de confusão de cada grupo; 6 . Principais achados dos estudos descritos; 7. Estimativas de variabilidade aleatória dos dados dos principais achados; 8 . Efeitos adversos relatados; 9. Características dos pacientes perdidos descritas; 10. Intervalos de confiança ou valores de $p$ para os principais achados; 11 . Sujeitos chamados para participar foram representativos da população; 12. Sujeitos preparados para participar foram representativos da população; 13. Equipes, lugares e instalações representativos do tratamento que a maioria dos pacientes recebem; 14 . Cegamento dos pacientes ao tipo de intervenção que receberam; 15. Cegamento dos mensuradores dos desfechos; 16. Análise exploratória; 17. Mesmo tempo de seguimento; 18. Testes estatísticos adequados; 19. Aderência à intervenção; 20. Medidas dos desfechos validadas e confiáveis; 21. Pacientes em diferentes grupos de intervenção foram recrutados da mesma população; 22. Pacientes em diferentes grupos de intervenção foram recrutados no mesmo período de tempo; 23 . Sujeitos do estudo randomizados para grupos de intervenções; 24 . Intervenção randomizada foi oculta para os pacientes e equipe; 25. Ajuste adequado dos fatores de confusão nas análises; 26. Perdas dos pacientes levadas em conta na análise e 27. Poder para detectar efeito clinicamente importante. 


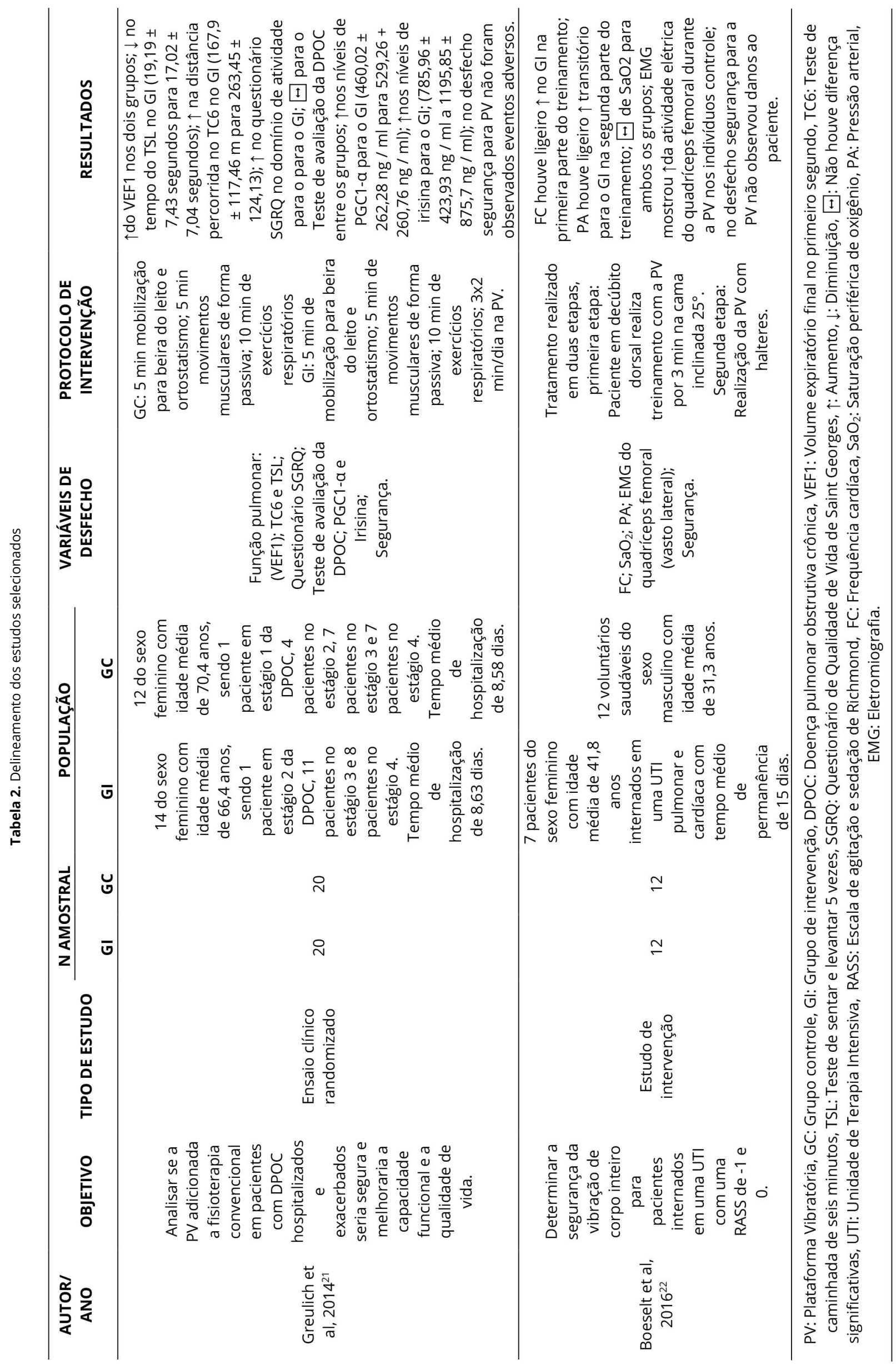




\section{Características dos estudos}

As características gerais estão sintetizadas na Tabela 2, tais como os procedimentos metodológicos e os principais resultados. Em relação a metodologia ambos estudos foram controlados, sendo 1 um ensaio clínico e o outro um estudo de intervenção não randomizado. $O$ tamanho amostral variou entre 24 a 40 pacientes totalizando 64 indivíduos, de ambos os sexos, com média de idade de 52,4 anos. Ambos avaliaram o uso da PV quanto a questões de segurança, variando quanto a análise da viabilidade (diagnósticos variados), e da capacidade funcional e qualidade de vida (diagnóstico de DPOC).

Em relação ao desfecho de segurança não foram observados a incidência de efeitos adversos, além de também não apresentar alterações significativas dos parâmetros clínicos representados pelos sinais vitais dos indivíduos submetidos a aplicação da PV. Quanto ao aspecto da funcionalidade foi observado o aumento na distância percorrida no teste de caminhada de 6 minutos (TC6) - 167,9 \pm 117,46 m para 263,45 \pm 124,13 - e diminuição do tempo no teste de sentar e levantar (TSL) - 19,19 \pm 7,43 segundos para 17,02 $\pm 7,04$ segundos - , além do aumento dos níveis de irisina no grupo de intervenção que foram submetidos a PV. Houve também correlação positiva da qualidade de vida observada no questionário de Saint Georges (SGRQ). As sessões de exposição na PV foram com um tempo médio de 4,5 minutos. Na Tabela 3 encontram-se os parâmetros utilizados em cada estudo. Tais características tiveram divergências quanto ao tempo de exposição.

Tabela 3. Características da plataforma vibratória nos estudos analisados

\begin{tabular}{ccc}
\hline & Greulich et al, 2014 & Boeselt et al, 2016 \\
\hline Modelo & GalileoTM & GalileoTM \\
\hline Frequência $(\mathbf{H z})$ & $26 \mathrm{~Hz}$ & $24 \mathrm{~Hz}$ \\
\hline Tempo de exposição & $3 \times 2 \mathrm{~min}$ & $4 \mathrm{~min}$ \\
\hline
\end{tabular}

\section{Discussão}

Foi possível observar que a PV parece ser segura e promover melhora na funcionalidade em pacientes adultos hospitalizados, visto que não foram observados a incidência de efeitos adversos, além de também não apresentarem alterações significativas dos sinais vitais. Além disso, houve uma melhora na distância percorrida no TC6 e diminuição do tempo no TSL, aumento dos níveis séricos de irisina marcadores de atividade muscular e uma melhora na qualidade de vida. É importante salientar que para se alcançar os objetivos funcionais propostos, deve-se superar o desafio de escolher estratégias que representem um mínimo estresse cardiorrespiratório e neuromuscular, sem ultrapassar os limites fisiológicos para essa população. Isso é um grande desafio na prática diária na área hospitalar, já que muitas vezes os pacientes são estereotipados e são realizados subdoses de exercício sem a programação de uma meta definida 23,24 .

Diversos estudos com PV já se mostraram benéficos em desfechos e populações variadas como aumento de força muscular, equilíbrio, capacidade funcional, manutenção da massa óssea e em idosos, pacientes com DPOC, Esclerose Múltipla e Acidente Vascular Encefálico, apesar de no ambiente hospitalar termos encontrado poucos estudos $25-29$.

Dessa forma quando se pensa na segurança desses e de qualquer outro paciente, os sinais vitais apresentam-se como importantes sinalizadores à tolerância terapêutica ${ }^{30}$. Marcadores como frequência cardíaca (FC); pressão arterial (PA) e saturação periférica de oxigênio (SpO2), não devem exceder limites estabelecidos nos critérios de segurança 31 . 
Uma clássica diretriz publicada em 2007 por Stiller, que aborda a segurança sobre mobilização de pacientes críticos, sugere a manutenção da FC entre 50 a 60\% do seu valor máximo predito durante a mobilização ${ }^{31}$. Tanto elevações acima desse percentual, quanto quedas da FC após o início de qualquer atividade, podem evidenciar a incompatibilidade física para a intensidade ou para o tipo de exercício empregado ${ }^{32}$.

No trabalho de Boeselt et. al. ${ }^{22}$ avaliado nesse referido artigo, verificou-se médias de FC em valores extremamente próximos aos sugeridos por Stiller, tanto no grupo que realizou PV isoladamente, quanto no grupo que realizou PV associada ao uso de halteres. Sabe-se que diversos fatores podem contribuir para um maior valor basal e instabilidade da FC em pacientes graves, portanto seu comportamento deve ser levado em consideração juntamente a outros aspectos pelo terapeuta no momento da abordagem $29,33,34$.

Com relação aos demais sinais vitais, só foi possível observar oscilações da SpO2 e da pressão arterial diastólica no grupo de pacientes críticos, que foram submetidos ao uso da PV com halteres. Embora presentes, essas alterações em nenhum momento extrapolaram os critérios definidos por Stiller, que aponta quedas de $\mathrm{SpO} 2>4 \%$ ou alterações na PA > 20\% como preocupantes ${ }^{31}$.

Assim sendo, no estudo de Boeselt et al.22 nenhum dos sinais vitais avaliados apresentou comportamento indesejado; entretanto, essas respostas tidas como satisfatórias, poderiam ser justificadas por uma estimulação motora aquém do necessário. Para contrapor essa teoria, foi realizada uma eletromiografia em um dos voluntários, sob as mesmas condições de PV, demonstrando claramente uma atividade aumentada do músculo quadríceps femoral. Essa comprovação pôde nesse caso, ratificar a execução e efetividade da técnica em questão, sem prejuízo da FC, PA e SpO2.

O segundo trabalho examinado pelo nosso estudo foi o de Greulich e colaboradores ${ }^{21}$. Nesse caso foi possível concluir que pacientes hospitalizados com
DPOC exacerbada se beneficiaram de um programa de fisioterapia aliado ao uso da PV. Tanto a segurança quanto otimizações clínicas foram destacadas positivamente. A melhora do desempenho físico vista no TC6 e no TSL, pode justificar-se por adaptações neuromorfológicas causadas pela PV. As vibrações promovem sincronização das unidades motoras, otimização do processo de estiramento e inibição da musculatura antagonista, que possivelmente favoreceriam o restabelecimento da questão funcional 35 .

Corroborando com o uso da PV, as elevações dos níveis séricos de irisina reforçaram as vantagens da PV vistas no artigo de Greulich²1. Essa importante citocina é secretada pelo músculo esquelético em decorrência da sua ativação, e está ligada a uma série de efeitos positivos sobre o metabolismo energético. Portanto, quanto mais associada a liberação de irisina, mais eficaz parece ser o exercício físico em questão ${ }^{36}$.

Por fim, em consonância com a melhora física e clínica, ainda no trabalho de Greulich ${ }^{21}$ pôde-se perceber uma correlação positiva da qualidade de vida observada no questionário de SGRQ e no CAT-Score. Constatou-se que os pacientes que realizaram PV melhoraram substancialmente no domínio "atividade", refletindo sobretudo no CAT-score uma tendência de melhora na qualidade de vida. Assim como no trabalho de Boeselt르, não foram encontrados efeitos adversos atrelados ao uso da PV.

Quando o assunto é segurança e funcionalidade em pacientes hospitalizados, é importante destacar que a escolha isolada da PV deve ser o bastante. Fatores variados como intensidade, duração, volume, frequência, dentre outros; são cruciais para o sucesso de qualquer terapêutica relacionada ao exercício físico e devem ser pensados de forma individualizada, respeitando as peculiaridades de cada paciente. No entanto, embora pareça ser uma técnica viável, devese salientar que existe um tempo para sua aplicação e um custo envolvido, o que limita sua aplicação no ambiente hospitalar, reforçando a importância das técnicas já atualmente aplicadas pelo terapeuta. 
Existem algumas limitações na presente revisão que devem ser consideradas. Primeiramente o pequeno número de estudos encontrados, além dos diferentes tipos de protocolos aplicados sem o detalhamento necessário de parâmetros e pôr fim a qualidade metodológica que apresentaram os trabalhos.

\section{Conclusão}

Nós concluímos que a PV parece ser viável e segura, podendo trazer efeitos favoráveis na funcionalidade para o tratamento em pacientes adultos hospitalizados, como uma estratégia alternativa para a reabilitação de forma precoce. Contudo, ainda não podemos afirmar que tal terapia é efetiva, devido à escassez de estudos com a modalidade PV na população hospitalizada. Assim, é necessário a realização de novos ensaios clínicos com melhor rigor metodológico nessa população.

\section{Contribuições dos autores}

Moura RF e Gomes VA participaram da concepção, delineamento, busca, interpretação dos resultados e redação do artigo científico. Santos ACN participou da concepção, delineamento e redação do artigo científico. Barbosa RM participou do delineamento, interpretação dos resultados e redação do artigo científico. Martinez BP da interpretação dos resultados e redação do artigo científico.

\section{Conflitos de interesses}

Nenhum conflito financeiro, legal ou político envolvendo terceiros (governo, empresas e fundações privadas, etc.) foi declarado para nenhum aspecto do trabalho submetido (incluindo, mas não se limitando a subvenções e financiamentos, participação em conselho consultivo, desenho de estudo, preparação de manuscrito, análise estatística, etc.).

\section{Referências}

1. Valentin A. The importance of risk reduction in critically ill patients. Curr Opin Crit Care. 2010;16(5):482-6. doi: 10.1097/ MCC.0b013e32833cb861

2. Sarmento GJV. O ABC da fisioterapia respiratória. 2ed. São Paulo: Manole; 2015
3. Parry SM, Puthucheary ZA. The impact of extended bed rest on the musculoskeletal system in the critical care environment. Extrem Physiol Med. 2015;4:16. doi: 10.1186/s13728-015-0036-7

4. Suesada MM, Martins MA, Carvalho CRF. Effect of short-term hospitalization on functional capacity in patients not restricted to bed. Am J Phys Med Rehabil. 2007;86(6):455-62. doi: 10.1097/ PHM.0b013e31805b7566

5. Hermans G, Van Mechelen H, Clerckx B, Vanhullebusch T, Mesotten D, Wilmer A et al. Acute outcomes and 1-year mortality of ICU-acquired weakness: a cohort and propensity matched analysis. Am J Respir Crit Care Med. 2014;190(4):410-20. doi: 10.1164/rccm.201312-22570C

6. França EET, Ferrari F, Maciel F, Gonçalves AC, Valois M. Mobilização no paciente crítico: Procedimentos e Evidências. In: Guimarães FS, Martins JÁ, organizadores. PROFISIO Programa de Atualização em Fisioterapia em Terapia Intensiva Adulto. 1.ed. Porto Alegre: Artmed/Panamericana; 2010. p. 73-106.

7. Gosselink R, Bott J, Johnson M, Dean E, Nava S, Norrenberg $M$ et al. Physiotherapy for adult patients with critical illness: recommendations of the European Respiratory Society and European Society of Intensive Care Medicine Task Force on Physiotherapy for Critically III Patients. Intensive Care Med. 2008;34(7):1188-99. doi: 10.1007/s00134-008-1026-7

8. Hirschhorn AD, Richards D, Mungovan SF, Morris NR, Adams L. Supervised moderate intensity exercise imporves distance walked at hospital discharge following coronary artery bypass graft surgery: a randomised controlled trial. Heart Lung Circ. 2008;17(2):129-38. doi: 10.1016/j.hlc.2007.09.004

9. Silva APP, Maynard K, Cruz MR. Efeitos da fisioterapia motora em pacientes críticos: revisão de literatura. Rev Bras Ter Intensiva. 2010;22(1):85-91. doi: 10.1590/S0103-507X2010000100014

10. Sachetti A, Carpes MF, Dias AS, Sbruzzi G. Segurança no uso da eletroestimulação neuromuscular em pacientes graves: revisão sistemática. Rev Bras Ter Intensiva. 2018; 30(2):219-225. doi: 10.5935/0103-507x.20180036

11. Santos CRS, Vasconcelos J, Ledo APO. Benefícios e efeitos da utilização da prancha ortostática nas unidades de terapia intensiva. Pós Graduação em Fisioterapia Hospitalar. 2015;1-13.

12. Hashem MD, Parker AM, Needham DM. Early mobilization and Rehabilitation of Patients who are critically III. Chest. 2016; 150(3):722-31. doi: 10.1016/j.chest.2016.03.003

13. Runge M, Rehfeld G, Resnicek E. Balance training and exercise in geriatric patients. J. Musculoskel Neuron Interact. 2000;1(1):615.

14. Dolny DG, Reyes GFC. Whole body vibration exercise: training and benefits. Curr Sports Med Rep. 2008;7(3):152-7. doi: 10.1097/01.CSMR.0000319708.18052.a1 
15. Gerhardt F, Dumitrescu D, Gärtner C, Beccard R, Viethen $\mathrm{T}$, Kramer $\mathrm{T}$ et al. Oscillatory whole-body vibration improves exercise capacity and physical performance in pulmonary arterial hypertension: a randomised clinical study. Heart. 2017;103(8):592598. doi: 10.1136/heartjnl-2016-309852

16. Wollersheim T, Haas K, Wolf S, Mai K, Spies C, SteinhagenThiessen $\mathrm{E}$ et al. Whole-body vibration to prevent intensive care unit-acquired weakness: safety, feasibility, and metabolic response. Crit Care. 2017;21(1):9. doi: 10.1186/s13054-016-1576-y

17. Moher D, Liberati A, Tetzlaff J, Altman DG. Preferred reporting items for systematic reviews and meta-analyses: the PRISMA statement. PLoS Med 2009;6(7):e1000097. doi: 10.1371/journal. pmed.1000097

18. Ministério da Saúde. Secretaria de Ciência, Tecnologia e Insumos Estratégicos. Departamento de Ciência e Tecnologia. Coordenação-Geral de Gestão do Conhecimento. Diretrizes metodológicas: elaboração de revisão sistemática e metanálise de estudos observacionais comparativos sobre fatores de risco e prognóstico. Brasília: Ministério da Saúde; 2014.

19. Benjamin DR, Van de Water ATM, Peiris CL. Effects of exercise on diastasis of the rectus abdominis muscle in the antenatal and postnatal periods: a systematic review. Physiotherapy. 2014;100(1):1-8. doi: 10.1016/j.physio.2013.08.005

20. Ratcliffe E, Pickering S, McLean S, Lewis J. Is there a relationship between subacromial impingement syndrome and scapular orientation? A systematic review. Br J Sports Med. 2014;48(16):1251-6. doi: 10.1136/bjsports-2013-092389

21. Greulich T, Nell C, Koepke J, Fechtel J, Franke M, Schmeck $B$ et al. Benefits of whole body vibration training in patients hospitalised for COPD exacerbations-a randomized clinical trial. BMC Pulm Med. 2014;14: 60. doi: 10.1186/1471-2466-14-60

22. Boeselt T, Nell C, Kehr K, Holland A, Dresel M, Greulich T et al. Whole-body vibration therapy in intensive care patients: $A$ feasibility and safety study. J Rehabil Med. 2016;48(3):316-21. doi: 10.2340/16501977-2052

23. Nydahl P, Sricharoenchai T, Chandra S, Kundt FS, Huang M, Fischill $M$ et al. Safety of Patient Mobilization and Rehabilitation in the Intensive Care Unit. Systematic Review with MetaAnalysis. Ann Am Thorac Soc. 2017;14(5):766-777. doi: 10.1513/ AnnalsATS.201611-843SR

24. Doiron KA, Hoffmann TC, Beller EM. Early intervention (mobilization or active exercise) for critically ill adults in the intensive care unit. Cochrane Database Syst Rev. 2018;27;3(3):CD010754. doi: 10.1002/14651858.CD010754.pub2

25. Ritzmann R, Gollhofer A, Kramer A. The influence of vibration type, frequency, body position and additional load on the neuromuscular activity during whole body vibration. Eur J Appl Physiol. 2013;113(1):1-11. doi: 10.1007/s00421-012-2402-0
26. Slatkovska L, Alibhai SMH, Beyene J, Cheung AM. Effect of whole-body vibration on BMD: a systematic review and metaanalysis. Osteoporos Int. 2010; 21(12): 1969-80. doi: $10.1007 /$ s00198-010-1228-z

27. Lam FMH, Lau RWK, Chung RCK, Pang MYC. The effect of whole body vibration on balance, mobility and falls in older adults: a systematic review and meta-analysis. Maturitas. 2012;72(3):20613. doi: 10.1016/j.maturitas.2012.04.009

28. Yang $X$, Zhou Y, Wang P, He C, He H. Effects of whole body vibration on pulmonary function, functional exercise capacity and quality of life in people with chronic obstructive pulmonary disease: a systematic review. Clin Rehabil. 2015;30(5):419-31. doi: 10.1177/0269215515589202

29. Chanou K, Gerodimos V, Karatrantou K, Jamurtas A. Wholebody vibration and rehabilitation of chronic diseases: a review of the literature. J Sports Sci Med. 2012;11(2):187-200.

30. Lee H, Ko YJ, Jung J, Choi AJ, Suh GY, Chung CR. Monitoring of potential safety events and vital signs during active mobilization of patients undergoing continuous renal replacement therapy in a medical intensive care unit. Blood Purification. 2016;42(1):83-90. doi: $\underline{10.1159 / 000446175}$

31. Stiller K. Safety issues that should be considered when mobilizing critically ill patients. Crit Care Clin. 2007;23(1):35-53. doi: 10.1016/j.ccc.2006.11.005

32. Stiller K, Phillips A. Safety aspects of mobilising acutely ill inpatients. Physiotherapy Theory and Practice. 2003;19(4):239257. doi: $10.1080 / 09593980390246751$

33. Broucqsault-Dédrie C, Jonckheere J, Jeanne M, Nseir S. Measurement of heart rate variability to asses pain in sedated critically ill patients: a prospective observational study. PLOS ONE. 2016;11(1):e0147720. doi: 10.1371/journal.pone.0147720

34. Samsudin MI, Liu N, Prabhakar SM, Chong SL, Lye WK, Koh ZX. A novel heart rate variability based risk prediction model for septic patients presenting to the emergency department. Medicine. 2018;97(23):e10866. doi: 10.1097/MD.0000000000010866

35. Plentz RDM, Sisto IR. Plataforma vibratória: mecanismos fisiológicos de ação e evidências científicas. In: Associação Brasileira de Fisioterapia Cardiorrespiratória e Fisioterapia em Terapia Intensiva; Martins JA, Dal Corso S, organizadores. PROFISIO Programa de Atualização em Fisioterapia Cardiovascular e Respiratória: Ciclo 4. Porto Alegre: Artmed Panamericana; 2018. p. 119-41. (Sistema de Educação Continuada a Distância, v. 3).

36. Moreno M, Moreno-Navarrete JM, Fernández-Real JM. Irisina: ¿transmisor de mensajes del Olimpo? [Irisin: a messenger from the gods?]. Clin Investig Arterioscler. 2014;26(3):140-6. doi: 10.1016/j.arteri.2013.11.002 